\title{
Multiparticle Bound-State Formation following a Quantum Quench to the One-Dimensional Bose Gas with Attractive Interactions
}

\author{
Lorenzo Piroli, ${ }^{1}$ Pasquale Calabrese, ${ }^{1}$ and Fabian H. L. Essler ${ }^{2}$ \\ ${ }^{1}$ SISSA and INFN, via Bonomea 265, 34136 Trieste, Italy \\ ${ }^{2}$ The Rudolf Peierls Centre for Theoretical Physics, Oxford University, Oxford OX1 3NP, United Kingdom \\ (Received 7 October 2015; revised manuscript received 21 January 2016; published 19 February 2016)

\begin{abstract}
We consider quantum quenches from an ideal Bose condensate to the Lieb-Liniger model with an arbitrary attractive interaction strength. We focus on the properties of the stationary state reached at late times after the quench. Using recently developed methods based on integrability, we obtain an exact description of the stationary state for a large number of bosons. A distinctive feature of this state is the presence of a hierarchy of multiparticle bound states. We determine the dependence of their densities on interaction strength and obtain an exact expression for the stationary value of the local pair correlation $g_{2}$. We discuss ramifications of our results for cold atom experiments.
\end{abstract}

DOI: 10.1103/PhysRevLett.116.070408

Integrable models have a long and venerable history of providing crucial points of reference that have greatly facilitated our understanding of interacting many-particle quantum systems. While integrable models are by definition special, recent advances in the field of ultracold atoms have made it possible to realize them to a good approximation experimentally [1-7]. Moreover, small deviations from integrability normally only lead to small changes in experimentally observable quantities.

One of the most striking features of quantum integrable models is that they typically feature hierarchies of bound states that often involve arbitrary numbers of elementary particles [8,9]. Such bound states have proved to be difficult to observe in, e.g., neutron scattering experiments on quantum spin chain materials, because their signatures in equilibrium dynamics are often small. Recent experimental advances in cold atomic gases have made it possible to observe nonequilibrium dynamics in isolated many-particle quantum systems in exquisite detail [10-20]. Integrable systems have again played a key role in these developments. In particular, following the theoretical proposal of Ref. [21], signatures of propagating two particle bound states following a local quench in an (almost) integrable system were successfully observed experimentally [16]. One of the most exciting aspects of nonequilibrium dynamics in integrable systems is that it allows one to realize new stable states of matter. One remarkable example is the so-called super Tonks-Girardeau gas, obtained at late times after quenching a Bose gas from an infinite repulsive interaction to an infinite attractive one $[11,22]$. This new state of matter has truly remarkable properties [22-27], exhibiting stronger correlations than the repulsive Tonks-Girardeau gas (which has also been probed experimentally [2]). In this Letter we investigate a protocol very similar to the super Tonks-Giradeau case, i.e., a quench from noninteracting to attractive Bose gas. We show that the stationary state reached at late times after the quench is a novel state of matter distinguished by a characteristic distribution of the densities of multiparticle bound states.

We consider a one-dimensional gas of $N$ bosons with attractive pointlike interaction, i.e., the Lieb-Liniger model [28]

$$
H=-\frac{\hbar^{2}}{2 m} \sum_{j=1}^{N} \frac{\partial^{2}}{\partial x_{j}^{2}}-2 \bar{c} \sum_{i<j} \delta\left(x_{i}-x_{j}\right) .
$$

Here, $\bar{c}>0$ is the interaction strength and $m$ the mass of the particles (atoms). The former is related to the effective 1D scattering length $a_{1 \mathrm{D}}$, which can be tuned experimentally via Feshbach resonances [29], by $\bar{c}=\hbar^{2} / m a_{1 \mathrm{D}}$. We consider a system of length $L$ with periodic boundary conditions, and ultimately are interested in the limit $L \rightarrow \infty$, while keeping the density of the gas $D=N / L$ fixed. For later convenience we define the dimensionless coupling constant $\gamma=\bar{c} / D$. In the following, we fix $\hbar=2 m=1$. Quantum quenches in the Lieb-Liniger model have been widely investigated in the literature [30-42], but have mainly focused on the repulsive regime. The Lieb-Liniger model is solvable by Bethe ansatz for any value of $\bar{c}$ [28]. The eigenstates (called Bethe states) $\Psi_{\left\{\lambda_{j}\right\}}$ are parametrized by sets of $N$ complex momenta $\left\{\lambda_{j}\right\}_{j=1}^{N}$, which satisfy the quantization conditions ("Bethe equations")

$$
e^{-i \lambda_{j} L}=\prod_{k \neq j}^{N} \frac{\lambda_{k}-\lambda_{j}-i \bar{c}}{\lambda_{k}-\lambda_{j}+i \bar{c}}, \quad j=1, \ldots, N .
$$

Bethe states are given by superposition of plane waves [28] $\Psi_{\left\{\lambda_{j}\right\}}\left(x_{1}, \ldots, x_{N}\right)=\sum_{P} A_{P} \prod_{j=1}^{N} e^{i \lambda_{P_{\ell}} x_{\ell}}$, where the sum is over all permutations $P$ of the rapidities $\left\{\lambda_{j}\right\}$ and the amplitudes are $A_{P}=\prod_{N \geq \ell>k \geq 1}\left\{1+\left[i \bar{c} \operatorname{sgn}\left(x_{\ell}-x_{k}\right) /\right.\right.$ $\left.\left.\left(\lambda_{P_{\ell}}-\lambda_{P_{k}}\right)\right]\right\}$. In the attractive regime, the solutions of Eq. (2) arrange themselves into patterns in the complex rapidity plane consisting of "strings" $[8,43]$. A general solution with $N$ rapidities will consist of $N_{j}$ strings of length $j$, where $N=\sum_{j} j N_{j}$. A single $j$ string takes the form 
$\lambda_{\alpha}^{j, a}=\lambda_{\alpha}^{j}+\frac{i \bar{c}}{2}(j+1-2 a)+i \delta^{j, a}, \quad a=1, \ldots, j$.

Here, $\alpha$ and $a$, respectively, label the string under consideration and the individual rapidities within that string, while $\delta^{j, a}$ are exponentially small deviations in the system size $L$. Following standard practice we will ignore these deviations. The string centers $\lambda_{\alpha}^{j}$ are real numbers and fulfill a generalized Pauli principle that imposes all string centers to be different for a given solution of Eq. (2). It follows from the Bethe ansatz form of the wave function that string solutions correspond to multiparticle bound states in the sense that the wave function decays exponentially with respect to the distance between any two particles in the bound state. The energy and momentum of an $N$-particle Bethe state consisting of $N_{j}$ strings of length $j$ are

$$
K=\sum_{(j, \alpha)} j \lambda_{\alpha}^{j}, \quad E=\sum_{(j, \alpha)} j\left(\lambda_{\alpha}^{j}\right)^{2}-\frac{\bar{c}^{2}}{12} j\left(j^{2}-1\right) .
$$

Equation (4) shows that $\lambda_{\alpha}^{j}$ is the contribution of each particle in a $j$-particle bound state to the total momentum. The ground state has zero momentum and consists of a single $N$-string [8]. While the thermodynamic limit in thermal equilibrium does not exist [8], correlation functions can be calculated at zero density [44]. Crucially, in the quantum quench context of interest here, the infinite volume limit at fixed particle density does exist. In this limit macrostates can be described in complete analogy to the standard finitetemperature formalism [8] by particle and hole densities $\left\{\rho_{n}(\lambda)\right\},\left\{\rho_{n}^{h}(\lambda)\right\}$. In particular, $\rho_{n}(\lambda)$ gives the distribution of $n$-string centers of a macrostate which, in the thermodynamic limit, form a dense set on the real line. Similarly, $\rho_{n}^{h}(\lambda)$ is the distribution of holes of $n$-string centers. The latter is analogous to the distribution of holes (i.e., unoccupied states) known from the ideal Fermi gas at finite temperature. While in the case of free fermions it is trivially related to the Fermi-Dirac distribution, in the interacting case we are considering here, the relation between $\rho_{n}(\lambda)$ and $\rho_{n}^{h}(\lambda)$ is more involved and derives from the Bethe equations (2) [45].

The quench protocol.-Our initial state is the ground state in the absence of interactions, i.e., the BEC state $[33,36]$. The evolution for $t>0$ is governed by the attractive Lieb-Liniger Hamiltonian. At time $t$, the expectation value of any observable $O$ is given by (denoting energy eigenstates with $|\mu\rangle,|\nu\rangle$ )

$$
\langle\Psi(t)|O| \Psi(t)\rangle=\sum_{\mu, \nu}\left\langle\Psi_{0} \mid \mu\right\rangle\langle\mu|O| \nu\rangle\left\langle\nu \mid \Psi_{0}\right\rangle e^{i\left(E_{\mu}-E_{\nu}\right) t} .
$$

In the thermodynamic limit expectation values of local operators approach time independent values. These can be determined by the recently proposed quench action method [46]. The latter results in a particular set of particle and hole densities and a corresponding "representative eigenstate" $\left|\boldsymbol{\rho}_{\text {sp }}\right\rangle$ such that for local operators $\mathcal{O}$

$$
\lim _{t \rightarrow \infty}\langle\Psi(t)|\mathcal{O}| \Psi(t)\rangle=\left\langle\boldsymbol{\rho}_{\mathrm{sp}}|\mathcal{O}| \boldsymbol{\rho}_{\mathrm{sp}}\right\rangle .
$$

The state $\left|\boldsymbol{\rho}_{\mathrm{sp}}\right\rangle$, which depends on the initial state of the system $|\Psi(0)\rangle$, will be referred to as the stationary state towards which the system evolves in the sense of Eq. (6). In general the determination of the representative eigenstate is a nontrivial task and it has been carried out in only a few cases [36,47-51]. In the following, we report the exact analytical solution of this problem for the quench that we are considering.

The postquench stationary state. - As we already noted, the state $\left|\boldsymbol{\rho}_{\mathrm{sp}}\right\rangle$ is specified by sets of particle and hole densities $\left\{\rho_{n}(\lambda)\right\},\left\{\rho_{n}^{h}(\lambda)\right\}$. It is useful to work with the dimensionless variable $x=\lambda / \bar{c}$ instead of the rapidity $\lambda$, and with a slight abuse of notation we will keep the same symbol for functions of $\lambda$ and of $x$ when this does not generate confusion. We further define $\eta_{n}(x)=\rho_{n}^{h}(x) / \rho_{n}(x)$. Our result for the densities describing the stationary state is

$$
\begin{gathered}
\eta_{1}(x)=\frac{x^{2}\left[1+4 \tau+12 \tau^{2}+(5+16 \tau) x^{2}+4 x^{4}\right]}{4 \tau^{2}\left(1+x^{2}\right)}, \\
\eta_{n}(x)=\frac{\eta_{n-1}\left(x+\frac{i}{2}\right) \eta_{n-1}\left(x-\frac{i}{2}\right)}{1+\eta_{n-2}(x)}-1, \quad n \geq 2, \\
\rho_{n}(x)=\frac{\tau}{4 \pi} \frac{\partial_{\tau} \eta_{n}^{-1}(x)}{1+\eta_{n}^{-1}(x)},
\end{gathered}
$$

where we have defined $\eta_{0}(x) \equiv 0$ and $\tau=1 / \gamma$. Note that a relation analogous to Eq. (9) was also found in the quench to the repulsive Lieb-Liniger model [36]. Given $\eta_{1}(x)$ in Eq. (7), Eqs. (8) and (9) provide all other densities specifying the stationary state. Indeed, using the relation $\rho_{n}^{h}(x)=\eta_{n}(x) \rho_{n}(x)$, one can readily see that $\rho_{n}(x)$ and $\rho_{n}^{h}(x)$ are written as rational functions (the actual expressions getting lengthier as $n$ increases).

The knowledge of the distributions $\rho_{n}(\lambda), \rho_{n}^{h}(\lambda)$ allows one, in principle, to compute the expectation value of all local observables in the postquench stationary state. We considered the experimentally measurable local pair correlation (in the following equation $\hat{\rho}$ is the density operator)

$$
g_{2}=\left\langle: \hat{\rho}^{2}(0):\right\rangle / D^{2} \text {. }
$$

The computation of Eq. (10) can be performed using the Hellmann-Feynman theorem [52] and results in

$$
\begin{aligned}
g_{2}= & \gamma^{2} \sum_{m=1}^{\infty} \int_{-\infty}^{\infty} \frac{d x}{2 \pi}\left[2 m x b_{m}(x) \frac{1}{1+\tilde{\eta}_{m}(x)}\right. \\
& \left.-2 \pi \tilde{\rho}_{m}(x)\left(2 m x^{2}-\frac{m\left(m^{2}-1\right)}{6}\right)\right],
\end{aligned}
$$

where the functions $b_{n}(x)$ are determined by

$b_{n}(x)=n x-\sum_{m=1}^{\infty} \int_{-\infty}^{\infty} d y \frac{1}{1+\tilde{\eta}_{m}(y)} b_{m}(y) \tilde{a}_{n m}(x-y)$.

Here, we defined $\tilde{\eta}_{n}(x)=\eta_{n}(x \bar{c}), \tilde{\rho}_{n}(x)=\rho_{n}(x \bar{c})$ [note that the computation of $g_{2}$ in Eq. (11) requires the knowledge of the distributions $\eta_{n}, \rho_{n}$ characterizing the 


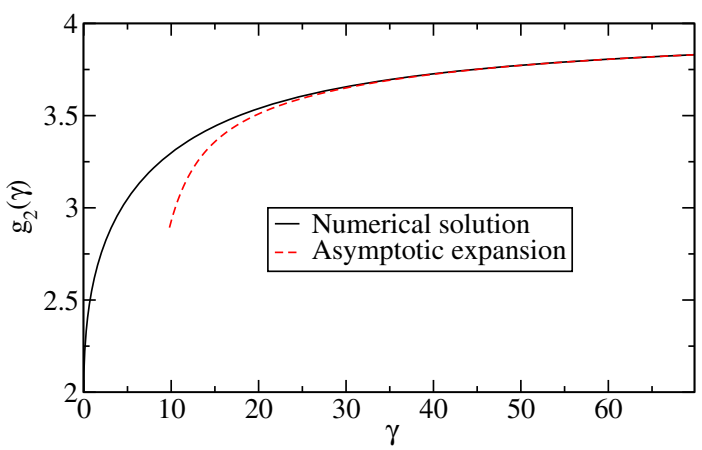

FIG. 1. Numerical value of $g_{2}(\gamma)$ given in Eq. (11) (solid line) and asymptotic analytical expansion as given in Eq. (14) (dashed line).

postquench stationary state]. Finally, in Eq. (12) we used $\tilde{a}_{n m}(x)=a_{n m}(x \bar{c})$, where

$$
\begin{aligned}
a_{n m}(\lambda)= & \left(1-\delta_{n m}\right) a_{|n-m|}(\lambda)+2 a_{|n-m|+2}(\lambda) \\
& +\ldots+2 a_{n+m-2}(\lambda)+a_{n+m}(\lambda),
\end{aligned}
$$

and $a_{n}(\lambda)=(2 / \pi n \bar{c})\left\{1 /\left[1+(2 \lambda / n \bar{c})^{2}\right]\right\}$. Equations (11) and (12) can be analytically solved for large $\gamma$ obtaining an expansion of $g_{2}$ in $1 / \gamma$ for $\gamma \rightarrow \infty$. Up to the third order it reads

$$
g_{2}(\gamma)=4-\frac{40}{3 \gamma}+\frac{344}{3 \gamma^{2}}-\frac{2656}{3 \gamma^{3}}+\mathcal{O}\left(\gamma^{-4}\right) .
$$

For generic $\gamma$ the sets of Eqs. (11) and (12) can be solved numerically, resulting in the curve shown in Fig. 1. The function $g_{2}(\gamma)$ displays two intriguing features. The first one is that it is discontinuous in $\gamma=0$. Indeed,

$$
\frac{\left\langle\mathrm{BEC}\left|: \hat{\rho}^{2}(0):\right| \mathrm{BEC}\right\rangle}{D^{2}}=1 \neq 2=\lim _{\gamma \rightarrow 0} g_{2}(\gamma) \text {. }
$$

The second one is that in the limit $\gamma \rightarrow \infty$ it tends to the finite value $g_{2}(\infty)=4$, cf. Eq. (14). This is, in contrast, with all other known stable situations, where the value of $g_{2}$ for infinite interaction is always vanishing. This is true, for example, in the repulsive regime at equilibrium (at finite or zero temperature) [53] and crucially in the attractive regime for the super-Tonks-Girardeau case, where $g_{2}$ is also vanishing for infinite interactions [22,23,25]. In the following, we argue that both of these behaviors can be ascribed to multiparticle bound state effects.

Bound state content and physical implications.-The most interesting property of our exact solution is that most of the particles after the quench form bound states. In Fig. 2 we display the particle densities for bound states involving up to four particles for two values of $\gamma$. We immediately see that bound particles outnumber unbound ones. To be more quantitative, we define the density $D_{n}$ and the energy $E_{n}$ of the particles forming $n$ strings as

$D_{n}=n \int_{-\infty}^{\infty} d \lambda \rho_{n}(\lambda), \quad E_{n}=\int_{-\infty}^{\infty} d \lambda \rho_{n}(\lambda) \varepsilon_{n}(\lambda)$,

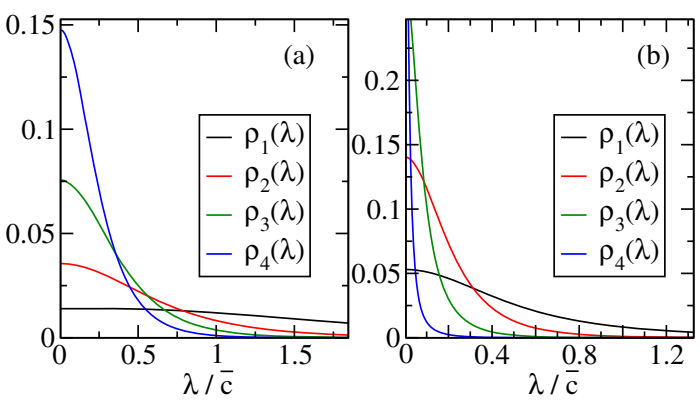

FIG. 2. Density distributions for the string centers $\rho_{n}(\lambda)$ for (a) $\gamma=0.5$ and (b) $\gamma=2$.

where $\varepsilon_{n}(\lambda)=n \lambda^{2}-\bar{c}^{2} n\left(n^{2}-1\right) / 12$. In terms of these quantities, the total density and energy are expressed as sums of contributions arising from $n$-particle bound states

$$
D=\sum_{n=1}^{\infty} D_{n}, \quad \frac{E}{L}=\sum_{n=1}^{\infty} E_{n} .
$$

In Fig. $3 D_{n}$ and $\left|E_{n}\right|$ are plotted for decreasing value of $\gamma=\bar{c} / D$ at a fixed density $D=1$. Figure 3 clearly shows that $n$ strings with $n \geq 2$, in fact, generally give the dominant contributions.

We now consider the dependence of the stationary state on $\gamma$ at fixed density. We note that the total energy is conserved during the quench and therefore is most easily calculated in the initial state using the Wick theorem, which gives

$$
\frac{E}{L}=-\gamma D^{3} .
$$

For large values of $\gamma$ the elementary bosons either remain unbound, or form two-particle bound states, cf. Fig. 3, while bound states involving more than 3 bosons do not play an important role. Moreover, it follows from Eq. (18) that $|E|$ is large, which implies that the binding energy is very high and we are dealing with tightly bound pairs of particles. These strongly affect physical properties of the stationary state even in the limit $\gamma \rightarrow \infty$. For example, the limiting value $g_{2}(\infty)$ can be imputed entirely to bound pairs [52]. As the value of $\gamma$

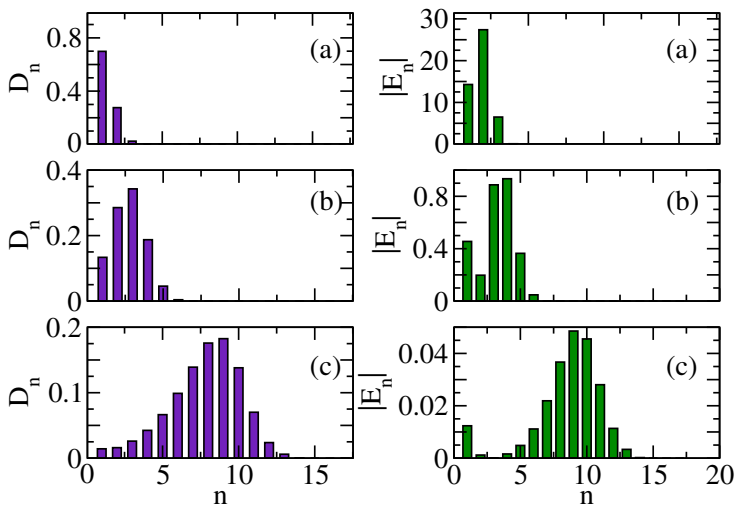

FIG. 3. Density $D_{n}$ and absolute value of the energy $\left|E_{n}\right|$ of the particles forming $n$ strings, as defined in Eq. (16), at fixed density $D=1$ and for (a) $\gamma=20$, (b) $\gamma=2$, and (c) $\gamma=0.2$. 
decreases, bound states of increasingly higher numbers of bosons become important. At the same time the magnitude of the total energy $|E|$ is seen to decrease, and the binding energy eventually approaches 0 for $\gamma \rightarrow 0$. Thus, in the limit $\gamma \rightarrow 0$, heuristically, the state after the quench is described by an infinitely populated bound state having zero binding energy. We have already alluded to the fact that $g_{2}(\gamma)$ exhibits a discontinuity at $\gamma=0$, which has its origin in the presence of multiparticle bound states for all positive $\gamma$. This is in marked contrast to the situation found for quantum quenches in the case of repulsive interactions [36]. We note that $\gamma=0$ is a point of nonanalyticity for the solution of Eqs. (11) and (12), making the limit $\gamma \rightarrow 0$ of $g_{2}(\gamma)$ in Eq. (15) difficult to compute [52].

Exact solution by the quench action approach.-We now sketch the derivation of the results presented above. The basic idea is to determine a representative eigenstate with the property that expectation values of local operators in this state match their stationary values reached at late times in the thermodynamic limit; cf. Eq. (6). It was shown in Ref. [46] that a state with this property can be constructed as the saddle point of the so-called quench action. The latter is given by

$$
S_{Q A}[\boldsymbol{\rho}]=2 S[\boldsymbol{\rho}]-S_{Y Y}[\boldsymbol{\rho}],
$$

where $\boldsymbol{\rho}$ generically indicates a state corresponding to the sets of densities $\left\{\rho_{n}\right\}$ and $\left\{\rho_{n}^{h}\right\}$ and where $S_{Y Y}$ is the YangYang entropy

$\frac{S_{Y Y}[\boldsymbol{\rho}]}{L}=\frac{1}{2} \sum_{n=1}^{\infty} \int_{-\infty}^{\infty} d \lambda\left[\rho_{n} \ln \left(1+\eta_{n}\right)+\rho_{n}^{h} \ln \left(1+\eta_{n}^{-1}\right)\right]$.

The dependence of $S_{Q A}$ on the initial state $\left|\Psi_{0}\right\rangle$ enters through $S[\boldsymbol{\rho}]=-\lim _{\mathrm{th}} \operatorname{Re} \ln \left\langle\Psi_{0} \mid \boldsymbol{\rho}\right\rangle$, where $\lim _{\mathrm{th}}$ denotes the thermodynamic limit $N, L \rightarrow \infty$ at fixed $D=N / L$. In our case $\left|\Psi_{0}\right\rangle$ is the BEC state, which has vanishing overlaps with nonparity invariant Bethe states [this is the reason of the overall factor 1/2 in Eq. (20)] [36]. The main ingredient in the quench action approach are the overlaps $\left\langle\Psi_{0} \mid \boldsymbol{\rho}\right\rangle$ between the Bethe states and the initial state. These are typically difficult to compute, but have been derived for the LiebLiniger model in Refs. [36,54] and applied to the attractive case in Ref. [37] (other overlaps are also known [55-57]). In our case the functional $S[\boldsymbol{\rho}]$ can be expressed as

$S[\boldsymbol{\rho}]=\frac{L D}{2}(\ln \gamma+1)-\frac{L}{2} \sum_{n=1}^{\infty} \int_{0}^{\infty} d \lambda \rho_{n}(\lambda) \ln W_{n}(\lambda)$,

where

$$
W_{n}(\lambda)=\frac{1}{\frac{\lambda^{2}}{\bar{c}^{2}}\left(\frac{\lambda^{2}}{\bar{c}^{2}}+\frac{n^{2}}{4}\right) \prod_{j=1}^{n-1}\left(\frac{\lambda^{2}}{\bar{c}^{2}}+\frac{j^{2}}{4}\right)^{2}} .
$$

The saddle point conditions specifying the representative state $\left|\boldsymbol{\rho}_{\mathrm{sp}}\right\rangle$ are

$$
\left.\frac{\partial S_{Q A}[\boldsymbol{\rho}]}{\partial \rho_{n}(\lambda)}\right|_{\rho=\boldsymbol{\rho}_{\mathrm{sp}}}=0, \quad n \geq 1,
$$

and take the form of coupled integral equations

$\ln \eta_{n}(\lambda)=-2 h n-\ln W_{n}(\lambda)+\sum_{m=1}^{\infty} a_{n m} * \ln \left(1+\eta_{m}^{-1}\right)(\lambda)$,

where we indicated the convolution between two functions with $f * g(\lambda)=\int_{-\infty}^{\infty} \mathrm{d} \mu f(\lambda-\mu) g(\mu)$ and where $a_{n m}(\lambda)$ is defined in Eq. (13). Here the Lagrange multiplier $h$ has been introduced to fix the total density $D$. The solution of Eq. (24) defines the distributions $\eta_{n}(\lambda)$ for the saddle point state $\left|\rho_{\mathrm{sp}}\right\rangle$. A second set of equations is obtained by taking the thermodynamic limit of the Bethe equations (2), cf. Refs. [44,52]

$\frac{n}{2 \pi}-\sum_{m=1}^{\infty} \int_{-\infty}^{\infty} d \lambda^{\prime} a_{n m}\left(\lambda-\lambda^{\prime}\right) \rho_{m}\left(\lambda^{\prime}\right)=\rho_{n}(\lambda)\left[1+\eta_{n}(\lambda)\right]$.

The sets (24), (25) of integral equations completely determine the saddle point particle and hole densities. Their solution is given by Eqs. (7), (8), and (9), with the relation $\tau=e^{h}$ [52].

Experimental signature of the multiparticle bound states.-Given that the quench from a BEC to a gas with attractive interactions is clearly experimentally realizable as a simple modification of the super Tonks-Girardeau gas [11], an obvious question is whether there are smoking gun signatures of our novel state of matter. A key feature of our steady state is the presence of several species of bound states, which in the Lieb-Liniger model are infinitely long lived as a consequence of integrability. It can be shown following Ref. [58] that different bound states have different group velocities. This fact suggests that in the stationary state the spreading of a local perturbation will exhibit several "light cones," associated with different kinds of bound states $[12,16,19,59]$. We expect the situation to be analogous to what is seen theoretically [21] and experimentally [16] after local quantum quenches in the Heisenberg $X X Z$ chain in equilibrium. A detailed analysis of a local quench in our steady state is, however, beyond the scope of this Letter. An important issue with regards to realizing our quench protocol in cold atom experiments is the size of three-body losses. These can be estimated from the three-body local correlation function $g_{3}$. The calculation of $g_{3}$ in the presence of bound states is a challenging task, and we hope that our work will motivate studies in this direction.

Conclusions.-We have considered quantum quenches in the one-dimensional Bose gas with attractive deltafunction interactions. In equilibrium, this model is known to be thermodynamically unstable, because it supports the formation of many-particle bound states with binding energies that scale like the third power of the number of atoms in the bound state. The initial state in our quench is a $\mathrm{BEC}$, and we consider time evolution by the Lieb-Liniger Hamiltonian with arbitrary attractive interaction strength. We have determined the exact steady state reached at late times after this quench. This stationary state is 
thermodynamically stable as a consequence of energy conservation. We have shown that this state is characterized by the presence of finite densities of multiparticle bound states involving different numbers of atoms. This composition, which automatically ensures thermodynamic stability, distinguishes the stationary state in a clear and qualitative way from other known stable states of the model. In particular, our state differs significantly from the super-Tonks-Girardeau gas, which is characterized by the absence of bound states [22-25]. A very interesting feature of our steady state is that the "dominant" species of multiparticle bound states can be changed by tuning the value of the interaction strength; cf. Fig. 3. We have shown that the structure of the steady state results in a value of $g_{2}$ between 2 and 4, cf. Fig. 1, which is very different from other known stable states in the Lieb-Liniger model. Finally, we have argued that these bound states can be revealed by observing the spreading of local perturbations imposed at late times after the initial quench.

This work was supported by the EPSRC under Grants No. EP/I032487/1 and No. EP/J014885/1 (F. H. L. E.) and the ERC under Starting Grant No. 279391 EDEQS (P. C. and F. H. L. E.). F. H. L. E. thanks SISSA for hospitality.

[1] I. Bloch, J. Dalibard, and W. Zwerger, Rev. Mod. Phys. 80, 885 (2008); M. A. Cazalilla, R. Citro, T. Giamarchi, E. Orignac, and M. Rigol, Rev. Mod. Phys. 83, 1405 (2011).

[2] T. Kinoshita, T. Wenger, and D. S. Weiss, Science 305, 1125 (2004); B. Paredes, A. Widera, V. Murg, O. Mandel, S. Fölling, I. Cirac, G. V. Shlyapnikov, T. W. Hänsch, and I. Bloch, Nature (London) 429, 277 (2004).

[3] T. Kinoshita, T. Wenger, and D. S. Weiss, Phys. Rev. Lett. 95, 190406 (2005).

[4] A. H. van Amerongen, J. J. P. van Es, P. Wicke, K. V. Kheruntsyan, and N. J. van Druten, Phys. Rev. Lett. 100, 090402 (2008).

[5] N. Fabbri, M. Panfil, D. Clément, L. Fallani, M. Inguscio, C. Fort, and J.-S. Caux, Phys. Rev. A 91, 043617 (2015); N. Fabbri, D. Clément, L. Fallani, C. Fort, and M. Inguscio, Phys. Rev. A 83, 031604 (2011); T. Jacqmin, J. Armijo, T. Berrada, K. V. Kheruntsyan, and I. Bouchoule, Phys. Rev. Lett. 106, 230405 (2011); F. Meinert, M. Panfil, M. J. Mark, K. Lauber, J.-S. Caux, and H.-C. Nägerl, Phys. Rev. Lett. 115, 085301 (2015).

[6] J. Simon, W. S. Bakr, R. Ma, M. E. Tai, P. M. Preiss, and M. Greiner, Nature (London) 472, 307 (2011).

[7] G. Pagano, M. Mancini, G. Cappellini, P. Lombardi, F. Schäfer, H. Hu, X.-J. Liu, J. Catani, C. Sias, M. Inguscio, and L. Fallani, Nat. Phys. 10, 198 (2014).

[8] M. Takahashi, Thermodynamics of One-Dimensional Solvable Models (Cambridge University Press, Cambridge, England, 1999).

[9] G. Mussardo, Statistical Field Theory: An Introduction to Exactly Solved Models in Statistical Physics (Oxford University Press, Oxford, 2009).

[10] T. Kinoshita, T. Wenger, and D. S. Weiss, Nature (London) 440, 900 (2006).
[11] E. Haller, M. Gustavsson, M. J. Mark, J. G. Danzl, R. Hart, G. Pupillo, and H. C. Nägerl, Science 325, 1224 (2009).

[12] M. Cheneau, P. Barmettler, D. Poletti, M. Endres, P. Schauß, T. Fukuhara, C. Gross, I. Bloch, C. Kollath, and S. Kuhr, Nature (London) 481, 484 (2012).

[13] M. Gring, M. Kuhnert, T. Langen, T. Kitagawa, B. Rauer, M. Schreitl, I. Mazets, D. A. Smith, E. Demler, and J. Schmiedmayer, Science 337, 1318 (2012).

[14] U. Schneider, L. Hackermüller, J. P. Ronzheimer, S. Will, S. Braun, T. Best, I. Bloch, E. Demler, S. Mandt, D. Rasch, and A. Rosch, Nat. Phys. 8, 213 (2012).

[15] F. Meinert, M. J. Mark, E. Kirilov, K. Lauber, P. Weinmann, A. J. Daley, and H.-C. Nägerl, Phys. Rev. Lett. 111, 053003 (2013).

[16] T. Fukuhara, P. Schauß, M. Endres, S. Hild, M. Cheneau, I. Bloch, and C. Gross, Nature (London) 502, 76 (2013).

[17] T. Langen, R. Geiger, M. Kuhnert, B. Rauer, and J. Schmiedmayer, Nat. Phys. 9, 640 (2013); T. Langen, S. Erne, R. Geiger, B. Rauer, T. Schweigler, M. Kuhnert, W. Rohringer, I. E. Mazets, T. Gasenzer, and J. Schmiedmayer, Science 348, 207 (2015).

[18] K. Agarwal, E. G. Dalla Torre, B. Rauer, T. Langen, J. Schmiedmayer, and E. Demler, Phys. Rev. Lett. 113, 190401 (2014).

[19] R. Geiger, T. Langen, I. E. Mazets, and J. Schmiedmayer, New J. Phys. 16, 053034 (2014).

[20] A. Polkovnikov, K. Sengupta, A. Silva, and M. Vengalattore, Rev. Mod. Phys. 83, 863 (2011).

[21] M. Ganahl, E. Rabel, F. H. L. Essler, and H. G. Evertz, Phys. Rev. Lett. 108, 077206 (2012).

[22] G. E. Astrakharchik, J. Boronat, J. Casulleras, and S. Giorgini, Phys. Rev. Lett. 95, 190407 (2005).

[23] M. T. Batchelor, M. Bortz, X. W. Guan, and N. Oelkers, J. Stat. Mech. (2005) L10001; S. Chen, L. Guan, X. Yin, Y. Hao, and X.-W. Guan, Phys. Rev. A 81, 031609 (2010).

[24] D. Muth and M. Fleischhauer, Phys. Rev. Lett. 105, 150403 (2010).

[25] M. Kormos, G. Mussardo, and A. Trombettoni, Phys. Rev. A 83, 013617 (2011).

[26] M. Panfil, J. De Nardis, and J.-S. Caux, Phys. Rev. Lett. 110, 125302 (2013).

[27] W. Tschischik and M. Haque, Phys. Rev. A 91, 053607 (2015).

[28] E. H. Lieb and W. Liniger, Phys. Rev. 130, 1605 (1963); E. H. Lieb, Phys. Rev. 130, 1616 (1963).

[29] S. Inouye, M. R. Andrews, J. Stenger, H.-J. Miesner, D. M. Stamper-Kurn, and W. Ketterle, Nature (London) 392, 151 (1998)

[30] V. Gritsev, T. Rostunov, and E. Demler, J. Stat. Mech. (2010) P05012.

[31] J. Mossel and J.-S. Caux, New J. Phys. 14, 075006 (2012).

[32] D. Iyer and N. Andrei, Phys. Rev. Lett. 109, 115304 (2012); D. Iyer, H. Guan, and N. Andrei, Phys. Rev. A 87, 053628 (2013).

[33] M. Kormos, A. Shashi, Y.-Z. Chou, J.-S. Caux, and A. Imambekov, Phys. Rev. B 88, 205131 (2013).

[34] B. Pozsgay, J. Stat. Mech. (2014) P10045.

[35] G. Mussardo, Phys. Rev. Lett. 111, 100401 (2013).

[36] J. De Nardis, B. Wouters, M. Brockmann, and J.-S. Caux, Phys. Rev. A 89, 033601 (2014).

[37] P. Calabrese and P. Le Doussal, J. Stat. Mech. (2014) P05004. 
[38] M. Kormos, M. Collura, and P. Calabrese, Phys. Rev. A 89, 013609 (2014); M. Collura, M. Kormos, and P. Calabrese, J. Stat. Mech. (2014) P01009; P. P. Mazza, M. Collura, M. Kormos, and P. Calabrese, J. Stat. Mech. (2014) P11016.

[39] J. De Nardis and J.-S. Caux, J. Stat. Mech. (2014) P12012.

[40] J. De Nardis, L. Piroli, and J.-S. Caux, J. Phys. A 48, 43FT01 (2015).

[41] R. van den Berg, B. Wouters, S. Eliëns, J. De Nardis, R. M. Konik, and J.-S. Caux, arXiv:1507.06339.

[42] G. Goldstein and N. Andrei, Phys. Rev. B 92, 155103 (2015).

[43] J. B. McGuire, J. Math. Phys. (N.Y.) 5, 622 (1964).

[44] P. Calabrese and J.-S. Caux, Phys. Rev. Lett. 98, 150403 (2007); J. Stat. Mech. (2007) P08032.

[45] V. E. Korepin, N. M. Bogoliubov, and A. G. Izergin, Quantum Inverse Scattering Method and Correlation Functions (Cambridge University Press, Cambridge, England, 1993).

[46] J.-S. Caux and F. H. L. Essler, Phys. Rev. Lett. 110, 257203 (2013).

[47] B. Wouters, J. De Nardis, M. Brockmann, D. Fioretto, M. Rigol, and J.-S. Caux, Phys. Rev. Lett. 113, 117202 (2014); M. Brockmann, B. Wouters, D. Fioretto, J. De Nardis, R. Vlijm, and J.-S. Caux, J. Stat. Mech. (2014) P12009.

[48] B. Pozsgay, M. Mestyán, M. A. Werner, M. Kormos, G. Zaránd, and G. Takács, Phys. Rev. Lett. 113, 117203
(2014); M. Mestyán, B. Pozsgay, G. Takács, and M. A. Werner, J. Stat. Mech. (2015) P04001.

[49] A. De Luca, G. Martelloni, and J. Viti, Phys. Rev. A 91, 021603 (2015).

[50] B. Bertini, D. Schuricht, and F. H. L. Essler, J. Stat. Mech. (2014) P10035.

[51] E. Ilievski, J. De Nardis, B. Wouters, J.-S. Caux, F. H. L. Essler, and T. Prosen, Phys. Rev. Lett. 115, 157201 (2015).

[52] L. Piroli, P. Calabrese, and F. H. L. Essler (to be published).

[53] D. M. Gangardt and G. V. Shlyapnikov, Phys. Rev. Lett. 90, 010401 (2003); K. V. Kheruntsyan, D. M. Gangardt, P. D. Drummond, and G. V. Shlyapnikov, Phys. Rev. Lett. 91, 040403 (2003).

[54] M. Brockmann, J. Stat. Mech. (2014) P05006; M. Brockmann, J. De Nardis, B. Wouters, and J.-S. Caux, J. Phys. A 47, 145003 (2014); J. Phys. A 47, 345003 (2014).

[55] B. Pozsgay, J. Stat. Mech. (2013) P10028; (2014) P06011.

[56] L. Piroli and P. Calabrese, J. Phys. A 47, 385003 (2014).

[57] P. P. Mazza, J.-M. Stéphan, E. Canovi, V. Alba, M. Brockmann, and M. Haque, J. Stat. Mech. 013104 (2016).

[58] L. Bonnes, F. H. L. Essler, and A. M. Läuchli, Phys. Rev. Lett. 113, 187203 (2014).

[59] P. Calabrese and J. Cardy, Phys. Rev. Lett. 96, 136801 (2006); J. Stat. Mech. (2007) P06008. 